AperTO - Archivio Istituzionale Open Access dell'Università di Torino

\title{
Walking in an immersive virtual reality
}

\section{This is the author's manuscript}

Original Citation:

Availability:

This version is available http://hdl.handle.net/2318/79891

since

Terms of use:

Open Access

Anyone can freely access the full text of works made available as "Open Access". Works made available under a Creative Commons license can be used according to the terms and conditions of said license. Use of all other works requires consent of the right holder (author or publisher) if not exempted from copyright protection by the applicable law. 


\title{
Walking in an Immersive Virtual Reality
}

\author{
Francesco MENEGONI ${ }^{\mathrm{a}}$, Giovanni ALBANI ${ }^{\mathrm{a}}$, Matteo BIGONI ${ }^{\mathrm{a}}$, Lorenzo PRIANO ${ }^{\mathrm{a}, \mathrm{b}}$, \\ Claudio TROTTI ${ }^{\mathrm{a}}$, Manuela GALLI ${ }^{\mathrm{c}}$ and Alessandro MAURO ${ }^{\mathrm{a}, \mathrm{b}}$ \\ ${ }^{a}$ Department of Neurology and Neurorehabilitation - IRCCS Istituto Auxologico \\ Italiano, Verbania, Italy \\ ${ }^{\mathrm{b}}$ Department of Neurosciences - Università di Torino, Italy \\ ${ }^{\mathrm{c}}$ Bioengineering Department - Politecnico di Milano, Italy
}

\begin{abstract}
The aim of this study was to investigate the effects on gait induced by a completely immersive Virtual Reality (VR) with and without perturbation. Ten healthy subjects were analyzed during over-ground walking in different conditions: standard gait, VR gait and perturbed VR gait. Results showed that subjects immersed in the virtual environment walked slowly, with decreased cadence $(-13 \%)$ and stride length $(-28 \%)$ as well as increased base of support in terms of step width $(+20 \%)$. The perturbation of the VR caused an interesting effect: many computed parameters, still away from the standard gait condition, improved if compared to the unperturbed VR condition. In conclusion, walking in VR leads to gait instability, which is less pronounced in presence of perturbation, probably due to the reweight of sensory inputs. This study could represent the first step for the application of the proposed VR environment to pathological subjects.
\end{abstract}

Keywords. Virtual reality, gait analysis, biomechanics, postural stability

\section{Introduction}

Much of our brain and nervous system is devoted to the processing of sensory input, in order to construct detailed representations of the external environment. This elaborate processing would be of limited value, however, unless we had a way to act upon the environment that we are sensing. In fact, visual, vestibular, and proprioceptive sensory information play a key role in postural and gait stability, and indeed the integration of this multisensory information is the basis for the control of body spatial orientation and movement [1].

Recently, the use of motion capture and Virtual Reality (VR) has been directed towards developing paradigms aimed at altering the contribution of vision during gait and posture and recording the effects, thus opening up many new research possibilities in behavioral neuroscience. In the last decade VR has been used to investigate the role of optic flow in human walking [2], to explore the effects of visual perturbation on gait [3-5], as well as to evaluate the effects of visual perturbation on static posture [6], but there are still many lacks: the effects of perturbation-free VR on gait and posture are still unknown, and "spontaneous gait" and free movement in VR environments are still undetermined [5]. Most importantly, at present there are no studies reporting a system able to merge the subject's virtual representation and the virtual environment.

The aim of the current study was to investigate the effects on gait induced by VR with and without visual perturbation. For this purpose a novel system that integrates a motion analysis laboratory and VR was developed and tested on healthy subjects. 


\section{Materials and Methods}

\subsection{Subjects}

Ten healthy subjects ( 5 females, 5 males) participated in this study (mean age: $33 \pm 7$ years). The study was approved by the local ethical committee and all subjects gave their informed consent.

\subsection{Experimental setup}

The peculiarity of our setup was the integration of different components: motion analysis laboratory, graphic workstation (Motion Builder ${ }^{\mathrm{TM}}$, Autodesk, CA) and HeadMounted Display (HMD) (Glasstron, Sony, JP). The motion analysis laboratory was equipped with an optoelectronic motion analysis system (Vicon 460, Vicon Motion Systems, Oxford, UK) with six cameras operating at $120 \mathrm{~Hz}$, two force platforms (Kistler, $\mathrm{CH}$ ), and a synchronized video system. The system was able to synchronously acquire the position of retroreflective passive markers (spherical, $14 \mathrm{~mm}$ diameter) placed on the subject, the ground reaction forces and the movies on the frontal and sagittal plane. Retroreflective markers were attached on each subject according to Vicon Plug-In Gait full-body marker set (Fig. 1).

A virtual laboratory environment was created in order to closely resemble the real laboratory with a human model (avatar) inside (Fig. 1 and Fig. 2). Subject position, measured by means of markers and optoelectronic system, was used to update the avatar position in real time, as well as to record subject's movements for offline analysis. The HMD was connected to the graphic workstation and was able to display the avatar point of view of the 3D virtual laboratory in real time (Fig. 2).

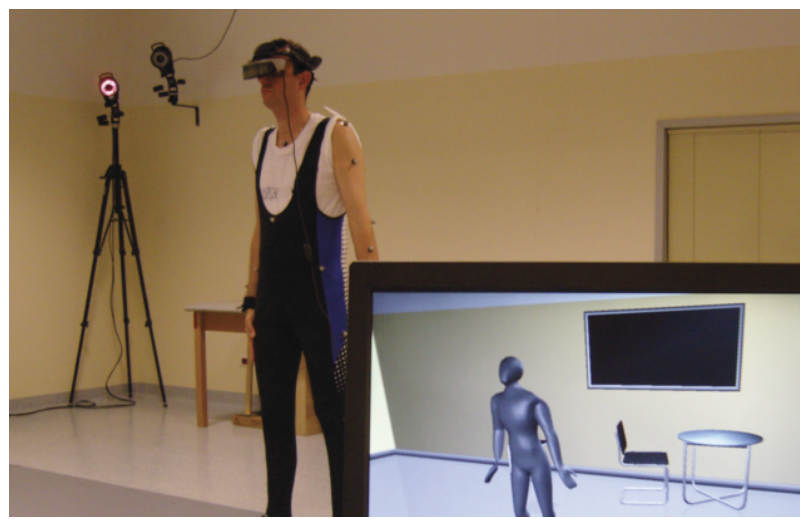

Figure 1. Subject wearing the HMD and markers was immersed in the movement analysis laboratory and consequently represented in the virtual laboratory. 


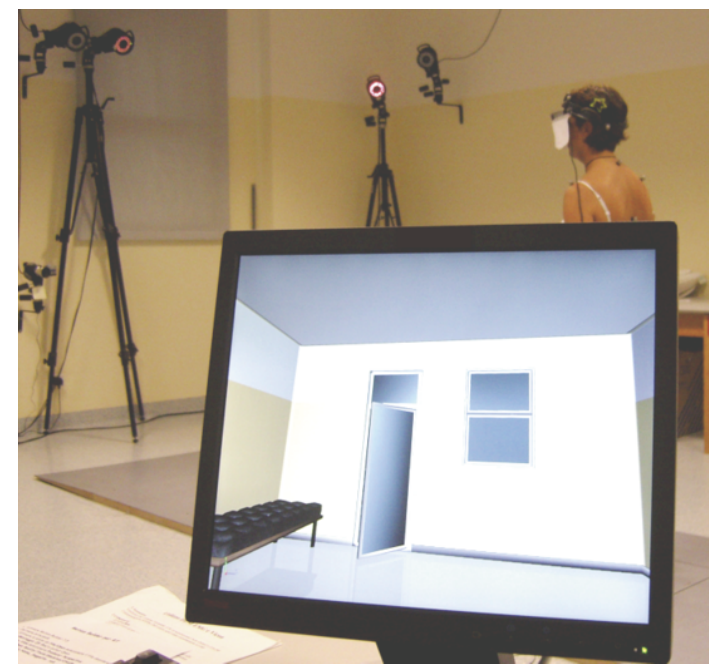

Figure 2. The image on the screen is the same of that one displayed on HMD and represents the virtual laboratory seen from the avatar perspective, while subject is walking.

Different conditions were tested: standard gait (SG), gait in VR (VRG) and gait in perturbed VR $\left(15^{\circ}\right.$ roll of the virtual laboratory - VRG15).

With regard to gait analysis trials data processing was computed using Workstation software. Following spatio-temporal parameters, usually related to gait instability, were then analyzed for each proposed condition: walking speed, cadence, stride length, step width, stance period (percent of gait cycle), and double support period (percent of cycle).

\subsection{Statistical analysis}

Gait parameters were compared among three proposed conditions using Friedman ANOVA for repeated measures $(p<0.05)$ followed by Wilcoxon paired test with Bonferroni's correction for the significance level $(p<0.017)$.

\section{Results}

All gait parameters were influenced by the VR conditions, as reported in Table 1. Subjects immersed in the virtual environment (VRG) walked slower than in standard condition ( $\mathrm{SG})$ : the statistically significant walking speed reduction $(-46 \%, \mathrm{p}=0.005)$ was caused by the combined effect of decreased cadence $(-14 \%, p=0.007)$ and diminution of stride length $(-33 \%, \mathrm{p}=0.005)$. At the same time, subjects showed an increased base of support in terms of step width $(+18 \%, \mathrm{p}=0.011)$, longer stance time $(+7 \%, \mathrm{p}=0.005)$, and increased double support $(+30 \%, \mathrm{p}=0.005)$.

The comparison between VRG15 and SG showed the same statistically significant differences found between VRG and SG. The successive comparison between VRG15 and VRG pointed out interesting results (Fig. 3). There were not statistically significant differences in the step width $(\mathrm{p}=0.83)$. Conversely all remaining parameters showed 
statistically significant improvements when compared to VRG: subjects walked faster $(+14 \%, \mathrm{p}=0.005)$, with increased cadence $(+7 \%, \mathrm{p}=0.01)$ and improved stride length

Table 1. Computed gait parameters in different conditions (values are expressed as mean plus/minus Standard Deviation).

\begin{tabular}{lrrrc}
\hline & SG & VRG & VRG15 & Friedman ANOVA \\
\hline Walking speed [m/s] & $1.07 \pm 0.16$ & $0.67 \pm 0.16$ & $0.77 \pm 0.17$ & $\mathrm{p}=0.001$ \\
Cadence [step/min] & $104.2 \pm 10.5$ & $90.3 \pm 7.8$ & $96.7 \pm 9.1$ & $\mathrm{p}=0.002$ \\
Stride length [m] & $1.23 \pm 0.10$ & $0.88 \pm 0.18$ & $0.95 \pm 0.18$ & $\mathrm{p}=0.001$ \\
Step width [m] & $0.15 \pm 0.04$ & $0.18 \pm 0.03$ & $0.18 \pm 0.04$ & $\mathrm{p}=0.002$ \\
$\quad$ Stance [\%] & $61.9 \pm 1.7$ & $66.6 \pm 3.1$ & $64.8 \pm 2.9$ & $\mathrm{p}=0.001$ \\
Double support [\%] & $24.1 \pm 3.4$ & $32.7 \pm 6.3$ & $29.4 \pm 5.9$ & $\mathrm{p}=0.001$ \\
\hline
\end{tabular}

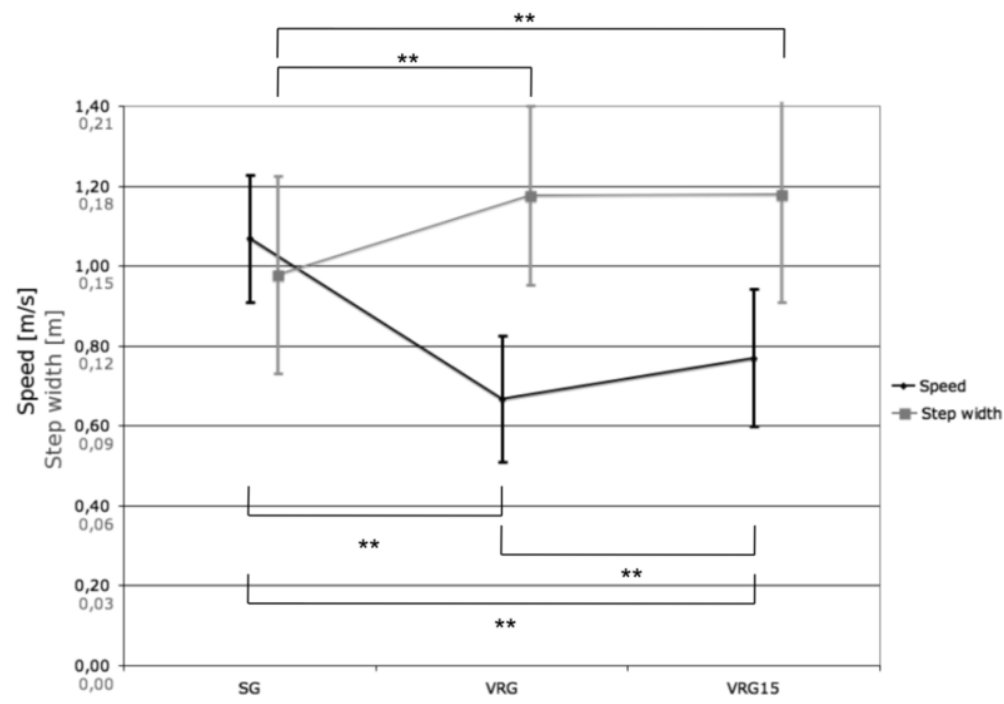

Figure 3. Walking speed (black line) and step width (grey line) reported in the three analyzed gait conditions $* * \mathrm{p}<0.01$.

$(+7 \%, \mathrm{p}=0.013)$. Stance time decreased $(-3 \%, \mathrm{p}=0.007)$ as well as double support time $(-10 \%, \mathrm{p}=0.005)$.

\section{Discussion and Conclusion}

The purpose of this study was to investigate the effects of immersion in virtual environment on gait. The system was designed to satisfy our aims and it overcomes the primary limitation reported in previous studies, related to the usage of the treadmill for gait analysis in VR. To authors knowledge this VR system is the first one that allows free gait, full offline analysis, a surrounding and convincing environment, and the vision of own body movements, an important feature in system with HMD [7].

The virtual environment we developed is a virtual laboratory very close to the real laboratory (dimensions, colors, objects) and thus we hypothesized that the 
performances of gait in VR would be comparable to those reported in real environment. Even more we provided an altered virtual laboratory $\left(15^{\circ}\right.$ roll VR) and we expected the perturbation would decrease gait performances.

Results about gait analysis show that consequences of immersion in the virtual environment (i.e.: VRG) are evident: subjects are not able to walk as they do in the real world. Specifically, decreased velocity and stride length, increased step width, increased stance time, and double support, have all been identified as markers of gait instability and are the basis of the characteristically 'cautious' gait pattern commonly observed in elderly people. These findings agree with those reported in previous studies about the less stable gait during treadmill locomotion in perturbed VR [4,5], but are not aligned with our expectations. Many could be the causes of the observed behavior: poor re-weight of sensory information, fear to freely walk without seeing obstacles, and/or absence of a preliminary training to VR.

As for gait in the perturbed virtual environment, we expected the weakest gait performance, but we observed an increased performance with respect to VRG. Previous studies have never tested a similar hypothesis thus other authors do not support this result; only Richards and colleagues reported that subjects walking on a treadmill respond to scene rotation $(30 \% \mathrm{~s}$ roll) by slowing down [3], but our experimental conditions were completely different. The cause of the observed behavior could be related to the potential better details related to the different viewing angle (e.g. floorwall corners, more furniture) as well as could be the result of the re-weight sensory inputs, with less weight accorded to perturbed vision and as a consequence the adoption of an automatic motor scheme, similar to gait pattern reported in eye-closed gait.

At the end of the analysis, the thesis that better explains our results is related to the different re-weight of sensory information, but a deeper investigation is needed to confirm it. In conclusion, this preliminary study reports that the system we developed is able to alter visual inputs and assess consequent effects on human movement. It is attractive for clinical application and we believe that VR technology provides an excellent tool for neuroscience research.

\section{References}

[1] M.E. Cinelli, A.E. Patla, Task-specific modulations of locomotor action parameters based on on-line visual information during collision avoidance with moving objects, Hum Mov Sci. 27 (2008), 513-31.

[2] W.H. Warren, B.A. Jr, Kay, W.D. Zosh, A.P. Duchon, S. Sahuc, Optic flow is used to control human walking, Nat Neurosci. 4 (2001), 213-6.

[3] J.T. Richards, Mulavara AP and Bloomberg JJ. Postural Stability During Treadmill Locomotion as a Function of the Visual Polarity and Rotation of a Three-Dimensional Virtual Environment. Presence 13 (2004), 371-384.

[4] Hollman JH, R.H. Brey, R.A. Robb, T.J. Bang, and K.R. Kaufman, Spatiotemporal gait deviations in a virtual reality environment, Gait Posture 23 (2006), 441-444.

[5] J.H. Hollman, R.H. Brey, T.J. Bang, and K.R. Kaufman, Does walking in a virtual environment induce unstable gait? An examination of vertical ground reaction forces, Gait Posture 26 (2007), 289-294.

[6] H. Akizuki, A. Uno, K. Arai, S. Morioka, S. Ohyama, S. Nishiike, K. Tamura, N. Takeda, Effects of immersion in virtual reality on postural control, Neurosci Lett. 279 (2005), 23-26.

[7] M.V. Sanchez-Vives, and M. Slater, From presence to consciousness through virtual reality. Nat.Rev.Neurosci. 6 (2005), 332-339. 